\title{
Spectral Theory of Taylor Vortices \\ Part II. Proof of Nonoscillation
}

\author{
C.-S. YIH
}

Communicated by C.C. LIN

Table of Contents

1. Introduction . . . . . . . . . . . . . . . . . . . 288

2. The Situation at $R=0 \ldots \ldots$. . . . . . . . . . . . . . . . . . . . . 288

3. The Situation at $\varepsilon=0 \ldots \ldots 291$

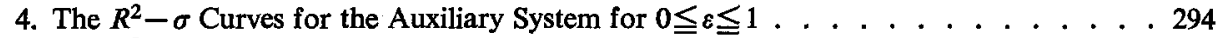

5. The $R^{2}-\sigma$ Curves for the Intermediate System for $0 \leqq \varepsilon \leqq 1$. . . . . . . . . . . . . . . 297

6. The $R^{2}-\sigma$ Curves for the Original System . . . . . . . . . . . . . . . . . . . . . 298

7. Recapitulation of Results for Positive $\sigma+\lambda^{2} \ldots \ldots$. . . . . . . . . . . . . 299

8. The Main Theorem . . . . . . . . . . . . . . . . . 299

\section{Introduction}

The principal objective of Part II of this paper is to prove the nonoscillation of Taylor vortices when the square of the circulation decreases outwards. The approach is briefly outlined in the following paragraph.

We first investigate the eigenvalues of $\sigma$ at $R=0$ for all three systems-the auxiliary system, the intermediate system, and the transitory system. These are all real, and they are independent of $\varepsilon$. Then for $\varepsilon=0$ we study the $R^{2}-\sigma$ curves for all three systems. These have the property that for any positive value of $R^{2}$ the values of $\sigma$ are all real. Finally we show that this property is retained in the range $0 \leqq \varepsilon \leqq 1$, which gives the required conclusion.

When sections and equations in Part I are referred to in this part, their numbers will be prefixed by "I". For instance, in Section I.2 the differential system governing Taylor vortices is given by (I.5), (I.6), and (I.7). The transitory system consists of (I.11), (I.12), and (I.7), the auxiliary system consists of (I.11), (I.12), and (I.14), and the intermediate system consists of (I.11), (I.12), (I.15), and (I.16). Apart from this modification, the notation in this part is the same as that in Part $I$.

\section{The Situation at $R=0$}

We can write $v^{\prime}=R v$ and convert (I.11) and (I.12) to

$$
\begin{gathered}
\left(L-\lambda^{2}-\sigma\right)\left(L-\lambda^{2}\right) u=4 \lambda^{2} R\left[1-\varepsilon B\left(1-\frac{1}{r^{2}}\right)\right] v^{\prime}, \\
\left(L-\lambda^{2}-\sigma\right) v^{\prime}=A R u,
\end{gathered}
$$


which are in fact the equations obtained immediately from the Navier-Stokes equations and the equation of continuity, before rewriting them as (I.11) and (I.12). For $R=0$, (1) and (2) become

$$
\begin{aligned}
\left(L-\lambda^{2}-\sigma\right)\left(L-\lambda^{2}\right) u & =0, \\
\left(L-\lambda^{2}-\sigma\right) v^{\prime} & =0 .
\end{aligned}
$$

Let us consider first the transitory system and the intermediate system for $R=0$, the former consisting of (3), (4), and

$$
\begin{gathered}
u(1)=D u(1)=0=u(b)=D u(b), \\
v^{\prime}(1)=0=v^{\prime}(b),
\end{gathered}
$$

and the latter consisting of (3), (4), and

$$
\begin{gathered}
u(1)=L u(1)=0=u(b)=D u(b), \\
v^{\prime}(1)=0=v^{\prime}(b) .
\end{gathered}
$$

For either system there are two sets of solutions. The first set (Set I) corresponds to $u=0$ and a nonzero $v^{\prime}$ satisfying (4) and (6) or (8). The second set (Set II) corresponds to $v^{\prime}=0$ and a nonzero $u$ satisfying (3) and (5) or (7). It can be established easily that the eigenvalues of one set are different from those of the other set, and that they are all real and negative. The eigenvalues of Set I are obtained by equating $\left(-\lambda^{2}-\sigma\right)^{1 / 2}$ with the roots of

$$
J_{1}(x) N_{1}(b x)-N_{1}(x) J_{1}(b x)=0
$$

where $J_{1}$ is the Bessel function and $N_{1}$ the Neumann function.

For the auxiliary system it is best to return to (I.10). For $R=0$ that equation becomes

$$
\left(L-\lambda^{2}-\sigma\right)^{2}\left(L-\lambda^{2}\right) v=0 .
$$

The boundary conditions for the auxiliary system can be written

$$
v(1)=L v(1)=L^{2} v(1)=0=v(b)=L v(b)=L^{2} v(b) .
$$

It is evident that the system consisting of (10) and (11) has eigenvalues of multiplicity two, and that they are exactly equal to the eigenvalues of Set I of the intermediate and original systems at $R=0$. We shall then consider each of these to be two identical simple eigenvalues for the auxiliary system: one belonging to Set I and the other to Set II. The eigenvalues of $\sigma$ of Set I for $R=0$ are therefore exactly the same for all three systems.

The simplicity of the eigenvalues of $\sigma$ of Set $I$ is obvious. We shall show the simplicity of the eigenvalues of $\sigma$ of Set II for all three systems. For Set II, the system consisting of (3) and (5) will be denoted by $Z$ (the $Z$ for zero, which is the value of $R$ ). The auxiliary system consisting of (3) and

$$
u(1)=L u(1)=0=u(b)=L u(b)
$$


will be denoted by $Z_{a}$. The intermediate system consisting of (3) and (7) will be denoted by $Z_{i}$. The system $Z_{a}$ can be written as

$$
\begin{gathered}
\left(L-\lambda^{2}-\sigma\right) Q=0, \\
Q(1)=0=Q(b)
\end{gathered}
$$

where $Q=\left(L-\lambda^{2}\right) u$. Now let

$$
u_{i}(1)=L u_{i}(1)=0=u_{i}(b) \quad(i=1 \text { or } 2),
$$

where $u_{1}$ corresponds to $\sigma_{1}$ and $u_{2}$ corresponds to $\sigma_{2}$, such that

and

$$
\left(L-\lambda^{2}-\sigma_{1}\right)\left(L-\lambda^{2}\right) u_{1}=0
$$

$$
\left(L-\lambda^{2}-\sigma_{2}\right)\left(L-\lambda^{2}\right) u_{2}=0 .
$$

Multiplying (16) by $r u_{2}$ and integrating between 1 and $b$, we have

$$
I_{m 2}+2 \lambda^{2} I_{m 1}+\lambda^{4} I_{m 0}-b D u_{2}(b) L u_{1}(b)=-\sigma_{1}\left(I_{m 1}+\lambda^{2} I_{m 0}\right),
$$

with the I's defined in (I.79). Similarly, we have

$$
I_{m 2}+2 \lambda^{2} I_{m 1}+\lambda^{4} I_{m 0}-b D u_{1}(b) L u_{2}(b)=-\sigma_{2}\left(I_{m 1}+\lambda^{2} I_{m 0}\right) .
$$

For $\sigma=0$ the sign of $u$ must be uniform except at the end points, where $u$ is zero. For otherwise there would be two points at which $Q$ is zero and between which $Q$ is of one sign; then multiplying (13), with $\sigma=0$, by $Q$ and integrating between these points, we would obtain the absurd result

$$
\int \frac{1}{r}\{D(r Q)\}^{2}+\lambda^{2} \int r Q^{2}=0 .
$$

Thus taking $u$ to be positive throughout, we must have a negative $D u(b)$ for $\sigma=0$, and hence a negative $L u(b)$, as can be seen from (18) by taking $\sigma=0$ and dropping the subscripts. We shall, then, start with

$$
b D u(b)=-1 .
$$

Taking the difference of (18) and (19) and letting $\sigma_{2}$ approach $\sigma_{1}$, we have

$$
\frac{d}{d \sigma} L u(b)=-I_{1}-\lambda^{2} I_{0}
$$

where $I_{1}$ and $I_{0}$ are defined by (I.38). As $\sigma$ decreases (to $\sigma_{1}^{\prime \prime}$, say) $L u(b)$ must increase to zero, for otherwise there would be no negative eigenvalues of $\sigma$, in contradiction to the fact that countably many negative eigenvalues of $\sigma$ exist. As $L u(b)$ passes zero and becomes positive, we can make $b L u(b)=1$ and relax the condition $b D u(b)=-1$. Then the difference of (18) and (19) gives

$$
\frac{d}{d \sigma} D u(b)=-I_{1}-\lambda^{2} I_{0}
$$

which states that as $\sigma$ decreases further, $D u(b)$ must increase. It must pass through zero, for otherwise there would be only one negative real eigenvalue $\sigma_{1}^{\prime \prime}$ for the 
system $Z_{a}$. We have thus reached the first eigenvalue $\sigma_{1}^{\prime}$ for the system $Z_{i}$. The process goes on, and we see that $\sigma_{n}^{\prime}$ separate $\sigma_{n}^{\prime \prime}$ and vice versa. Moreover (20) and (21) show that $\sigma_{n}^{\prime \prime}$ and $\sigma_{n}^{\prime}$ are simple for all positive integral values of $n$.

The arguments leading to Theorem 10 of Part I next show that there is at least one eigenvalue $\sigma_{n}$ of the System $Z$ between $\sigma_{n}^{\prime}$ and $\sigma_{n+1}^{\prime}$. The arguments leading to Theorem 11 of Part I then show that there is exactly one $\sigma_{n}$ between $\sigma_{n}^{\prime}$ and $\sigma_{n+1}^{\prime}$. Furthermore, by considering the system consisting of (3) and

$$
u(1)=0=u(b)=D u(b),
$$

and letting $\sigma$ decrease indefinitely from zero, one sees that $D u(1)$ vanishes at $\sigma=\sigma_{n}(n=1,2, \ldots)$ and that, from two equations similar to (18) and (19),

$$
\begin{aligned}
& \frac{d}{d \sigma} D u(1)=-\left(I_{1}+\lambda^{2} I_{0}\right) \quad \text { if } \quad L u(1)=1, \\
& \frac{d}{d \sigma} D u(1)=I_{1}+\lambda^{2} I_{0} \quad \text { if } \quad L u(1)=-1,
\end{aligned}
$$

either of which shows that the eigenvalues $\sigma_{n}$, corresponding to $D u(1)=0$, are simple. We summarize the results in two theorems.

Theorem 1. For $R^{2}=0$ and Set II, the auxiliary system has infinitely many eigenvalues $\sigma_{n}^{\prime \prime}$ of $\sigma$, which are independent of $\varepsilon$. These are simple and separated by the eigenvalues $\sigma_{n}^{\prime}$. They also separate the $\sigma_{n}^{\prime}$, which also are simple.

Theorem 2. For $R^{2}=0$ and Set $I I$, the original system has infinitely many real and negative eigenvalues $\sigma_{n}$ of $\sigma$ which are independent of $\varepsilon$. These are simple and separated by the eigenvalues $\sigma_{n}^{\prime}$ of the intermediate system. They also separate the $\sigma_{n}^{\prime}$.

Recall that the double eigenvalues $\sigma_{n}^{\prime \prime}$ are considered as two simple eigenvalues, one belonging to Set I and the other to Set II.

\section{The Situation at $\varepsilon=0$}

For clarity we shall now denote the eigenvalues of $\sigma$ at $R=0$ by $\sigma_{n 0}^{\prime \prime}, \sigma_{n 0}^{\prime}$, and $\sigma_{n 0}$ for the three systems, respectively. The eigenvalues $\sigma_{n 0}^{\prime \prime}, \sigma_{n 0}^{\prime}$, and $\sigma_{n 0}$ exist for all positive integral values of $n$, as we have seen. These are identical for Set $I$. For any positive real $R^{2}$ and for $\varepsilon=0$, the eigenvalues $\sigma_{n}^{\prime \prime}, \sigma_{n}^{\prime}$, and $\sigma_{n}$, if they exist, must be real, as can easily be shown by equations of the form (I.71) and (I.72). As we increase $R$ from zero, no two of the eigenvalues $\sigma_{n}^{\prime \prime}$ (or of $\sigma_{n}^{\prime}$ or $\sigma_{n}$ ) can merge and disappear from the real $R^{2}-\sigma$ plane, for otherwise there would be complex eigenvalues of $\sigma^{\prime \prime}$ (or of $\sigma^{\prime}$ or $\sigma$ ). Hence, not only do infinitely many real eigenvalues $\sigma_{n}^{\prime \prime}, \sigma_{n}^{\prime}$, and $\sigma_{n}$ exist for any positive $R^{2}$, but also the eigenvalues $\sigma_{n}^{\prime \prime}$ of Set I or Set II at any positive $R^{2}$ are in one-to-one correspondence with the eigenvalues $\sigma_{n 0}^{\prime \prime}$ of Set I or Set II, respectively. The same is true of the other two sequences $\sigma_{n}^{\prime}$ and $\sigma_{n}$.

At $\varepsilon=0$ the eigenvalues $\sigma_{n}^{\prime \prime}$ for the auxiliary system can be calculated directly. We note that the eigenfunction (for $u$ or $v$ ) is simply

$$
Z_{1}\left(k_{n} r\right) \text {, }
$$


which is a linear combination of $J_{1}\left(k_{n} r\right)$ and $N_{1}\left(k_{n} r\right)$ and which satisfies (11), provided $k_{n}$ is the $n$-th root of (9). Substituting $Z_{1}\left(k_{n}\right)$ for $v$ in

$$
\left(L-\lambda^{2}-\sigma\right)^{2}\left(L-\lambda^{2}\right) v=4 \lambda^{2} R^{2} A v,
$$

which is (I.10) with $\varepsilon=0$, we have, upon writing $\sigma_{n}^{\prime \prime}$ for the $n$-th eigenvalue of $\sigma$ (of Set I or Set II, respectively) for the auxiliary system,

$$
\left(\sigma_{n}^{\prime \prime}+\lambda^{2}+k_{n}^{2}\right)^{2}\left(\lambda^{2}+k_{n}^{2}\right)=-4 \lambda^{2} R^{2} A
$$

or

$$
\sigma_{n}^{\prime \prime}=-k_{n}^{2}-\lambda^{2} \pm\left(\frac{-4 A R^{2} \lambda^{2}}{k_{n}^{2}+\lambda^{2}}\right)^{1 / 2},
$$

the positive sign before the radical being associated with Set $I$ and the negative sign with Set II.

The graphs for (23) are a series of parabolas touching the axis $R=0$ at the points

$$
\sigma=\sigma_{n 0}^{\prime \prime}=-k_{n}^{2}-\lambda^{2} \text {. }
$$

We already know that for any of the three systems all the eigenvalues of $\sigma$ are real for $\varepsilon=0$ and $R^{2} \geqq 0$. To see how the $\sigma$-eigenvalues of one system separate those of a neighboring ${ }^{\star}$ one, we shall consider (I.73) to (I.76), but with the same $R^{2}$ and two different values of $\sigma$, say $\sigma_{1}$ in (I.73) and (I.74), and $\sigma_{2}$ in (I.75) and (I.76). By demanding that

$$
u(1)=0=L u(1)=v(1), \quad u(b)=0=v(b),
$$

we can, by a procedure strictly similar to that used to obtain (I.78) and (I.80), obtain

and

$$
\begin{gathered}
I_{m 2}+\left(2 \lambda^{2}+\sigma_{1}\right) I_{m 1}+\lambda^{2}\left(\lambda^{2}+\sigma_{1}\right) I_{m 0}-b D u_{2}(b) L u_{1}(b) \\
=-4 \lambda^{2} R^{2} A^{-1}\left[J_{m 1}+\left(\lambda^{2}+\sigma_{2}\right) J_{m 0}\right]
\end{gathered}
$$

$$
\begin{gathered}
I_{m 2}+\left(2 \lambda^{2}+\sigma_{2}\right) I_{m 1}+\lambda^{2}\left(\lambda^{2}+\sigma_{2}\right) I_{m 0}-b D u_{1}(b) L u_{2}(b) \\
=-4 \lambda^{2} R^{2} A^{-1}\left[J_{m 1}+\left(\lambda^{2}+\sigma_{1}\right) J_{m 0}\right]
\end{gathered}
$$

where the $I$ 's and the $J$ 's are defined by (I.79). Subtracting these results, we find, for $b D u(b)=-1$,

$$
\left(I_{m 1}+\lambda^{2} I_{m 0}-4 \lambda^{2} R^{2} A^{-1} J_{m 0}\right)\left(\sigma_{2}-\sigma_{1}\right)=L u_{1}(b)-L u_{2}(b),
$$

which in the limit yields

$$
\frac{d}{d \sigma} L u(b)=-\left(I_{1}+\lambda^{2} I_{0}-4 \lambda^{2} R^{2} A^{-1} J_{0}\right)
$$

with the $I$ 's and the $J_{0}$ defined by (I.38) and (I.19). We start with a $\sigma \geqq-\lambda^{2}$ for which $E_{1}^{\prime \prime}$ is greater than the $R^{2}$ under consideration. Then for the starting $\sigma$, we have a negative $D u(b)$ and a negative $L u(b)$, according to the development in Section I.6. Holding $b D u(b)$ at the value -1 and decreasing $\sigma$, we know that

$\star$ The auxiliary system is a neighboring system for the intermediate system, which is a neighboring system for the transitory system. 
as $\sigma$ reaches the eigenvalue $\sigma_{1}^{\prime \prime}$, which is known to exist, $L u(b)$ will increase to zero according to (28). As $\sigma$ decreases beyond $\sigma_{1}^{\prime \prime}, L u(b)$ is positive. It will reach $b^{-1}$, for otherwise $L u(b)$ would never reach infinity when $D u(b)$ is kept at $-b^{-1}$ and $\sigma$ decreases indefinitely; this would indicate that $\sigma_{1}^{\prime}$ did not exist and hence that, with $b D u(b)=-1$ as $\sigma$ decreases, $L u(b)$ would never again vanish, thus contradicting our knowledge that the eigenvalues $\sigma_{n}^{\prime \prime}$ exist. (Since $L u(b)$ will become positive, and since we can always multiply the eigenfunctions by a constant, the choice of the value 1 for $b L u(b)$ involves no difficulty.) Keeping $b L u(b)$ at 1 , we obtain from (26) and (27)

$$
\left(I_{m 1}+\lambda^{2} I_{m 0}-4 \lambda^{2} R^{2} A^{-1} J_{m 0}\right)\left(\sigma_{2}-\sigma_{1}\right)=-D u_{2}(b)+D u_{1}(b),
$$

which in the limit yields

$$
\frac{d}{d \sigma} D u(b)=-\left(I_{1}+\lambda^{2} I_{0}-4 \lambda^{2} R^{2} A^{-1} J_{0}\right)
$$

This says that $D u(b)$ will increase as $\sigma$ is decreased continuously. We know $\sigma_{1}^{\prime}$ exists. Thus when $\sigma$ reaches $\sigma_{1}^{\prime}, D u(b)$ is zero. By continuing the argument in this way we produce the eigenvalues $\sigma_{n}^{\prime \prime}$ and $\sigma_{n}^{\prime}$. It is evident that the two sets of eigenvalues separate each other, and from (28) and (29) (recalling that $A$ is negative) we see that not two of them in any one system can merge smoothly into each other if $R^{2} \geqq 0$, although there can be more than one mode at an eigenvalue pair $\left(R^{2}, \sigma\right)$, where two $R^{2}-\sigma$ curves for the same system intersect.

For clarity, we shall call any point in the $R^{2}-\sigma$ plane for which there is more than one mode a point of multiple modes, or simply a multiple point. At a multiple point two modes may or may not merge smoothly into each other. For instance, if $L u(b)$ is not zero (so that (29) holds) the point where the right-hand side of (29) vanishes is a multiple point where two modes merge smoothly into each other, if above or below that point there are two real (nonoscillatory) modes. For simplicity, we shall use the word "non-merging" for "not merging smoothly."

Thus we have

Theorem 3. For $\varepsilon=0$ and any positive $R^{2}$, the eigenvalues $\sigma_{n}^{\prime \prime}$ for the auxiliary system and the eigenvalues $\sigma_{n}^{\prime}$ for the intermediate system are real and non-merging, and the two sets separate each other.

Now consider, instead of (25), the boundary conditions

$$
u(1)=0=v(1), \quad u(b)=D u(b)=0=v(b),
$$

and let $\sigma$ decrease from some value for which $E_{1}^{\prime}$ is greater than the value of $R^{2}$ considered. A development similar to that leading to Theorem 3 now yields

Theorem 4. For $\varepsilon=0$ and any positive $R^{2}$, the eigenvalues $\sigma_{n}^{\prime}$ for the intermediate system and the eigenvalues $\sigma_{n}$ for the origin system (or starting system) are real and non-merging, and the two sets separate each other.

Note that for any of the three systems the $R^{2}-\sigma$ curves at $\varepsilon=0$ cannot have a maximum or minimum with positive $R^{2}$, for above such a maximum or below such a minimum $\sigma$ would be complex, and we know from (I.72) that $\sigma$ cannot be complex if $R^{2}$ is non-negative. The $n$-th $R^{2}-\sigma$ curve of the intermediate system 
for $\varepsilon=0$ then passes through $\sigma_{n 0}^{\prime}$ of Set I and $\sigma_{n 0}^{\prime}$ of Set II, joins smoothly below the $\sigma$-axis (because for any real finite $\sigma$ the value of $R^{2}$ cannot be negative infinite), and has two real values of $\sigma_{n}^{\prime}$ for $R^{2} \geqq 0$. Similarly, the $n$-th $R^{2}-\sigma$ curve of the starting system passes through $\sigma_{n 0}^{\prime}$ of Set I and $\sigma_{n 0}^{\prime}$ of Set II, joins smoothly below the $\sigma$-axis, and gives two real values for $\sigma_{n}$ for any $R^{2} \geqq 0$.

According to Theorem 3, through any point in the $R^{2}-\sigma$ plane where the auxiliary system has a double root, there must pass an $R^{2}-\sigma$ curve of the intermediate system, and vice versa. The same is true for the intermediate system and the starting system. This is true not merely for $\varepsilon=0$ but for nonzero $\varepsilon$ as well, for one can combine two modes to satisfy a new boundary condition. Recall that any point where two or more modes exist is called a multiple point. Any point representing an eigenvalue pair which is not a multiple point is called a simple point.

\section{The $R^{2}-\sigma$ Curves for the Auxiliary System for $0 \leqq \varepsilon \leqq 1$}

We recall that for any $\varepsilon$ in $0 \leqq \varepsilon \leqq 1$, the eigenvalues of $R^{2}$ for the auxiliary system are real if $\sigma$ is real, in virtue of (I.18). This means that the $R^{2}-\sigma$ curves for that system cannot have a maximum or a minimum in $\sigma$, to the right or left of which there would be complex eigenvalues of $R^{2}$.

We shall now show that the $R^{2}-\sigma$ curves mentioned above cannot have a maximum or minimum in $R^{2}$, so that all the eigenvalues of $\sigma$ for non-negative $R^{2}$, which are real for $\varepsilon=0$, remain real for $0<\varepsilon \leqq 1$. For the proof of this result, we again use the method of eigenvalue evolution.

Consider the adjoint generating system defined by

$$
\begin{gathered}
\left(L-\lambda^{2}-\sigma\right)\left(L-\lambda^{2}\right) \hat{u}=4 \lambda^{2} R^{2} \hat{v}, \\
\left(L-\lambda^{2}-\sigma\right) \hat{v}=A\left[1-\varepsilon B\left(1-\frac{1}{r^{2}}\right)\right] \hat{u},
\end{gathered}
$$

with the boundary conditions

$$
\hat{v}=\hat{u}=L \hat{u}=0 \text { at } r=1 \text {, and } \hat{u}=\hat{v}=0 \text { at } r=b \text {, }
$$

for any $\sigma$ or $R$. A sixth condition is to be applied at $r=b$. It is well-known that the eigenvalues of the system specified above are identical to those of the system to which it is adjoint.

Now consider the system

$$
\begin{gathered}
\left(L-\lambda^{2}-\sigma_{1}\right)\left(L-\lambda^{2}\right) \hat{u}_{1}=4 \lambda^{2} R_{1}^{2} \hat{v}_{1}, \\
\left(L-\lambda^{2}-\sigma_{1}\right) \hat{v}_{1}=A\left[1-\varepsilon B\left(1-\frac{1}{r^{2}}\right)\right] \hat{u}_{1},
\end{gathered}
$$

where $\hat{u}_{1}$ and $\hat{v}_{1}$ satisfy (33), together with the system

$$
\begin{gathered}
\left(L-\lambda^{2}-\sigma_{2}\right)\left(L-\lambda^{2}\right) u_{2}=4 \lambda^{2} R_{2}^{2}\left[1-\varepsilon B\left(1-\frac{1}{r^{2}}\right)\right] v_{2}, \\
\left(L-\lambda^{2}-\sigma_{2}\right) v_{2}=A u_{2},
\end{gathered}
$$

where $u_{2}$ and $v_{2}$ satisfy (I.7), with the exception of the condition $D u(b)=0$. 
Multiplying (34) by $r u_{2}$, integrating between 1 and $b$, and using (37) to evaluate $u_{2}$ on the right-hand side, we have

$$
\begin{gathered}
\hat{I}_{m 2}+\left(2 \lambda^{2}+\sigma_{1}\right) \hat{I}_{m 1}+\lambda^{2}\left(\lambda^{2}+\sigma_{1}\right) \hat{I}_{m 0}-b D u_{2}(b) L \hat{u}_{1}(b) \\
=-4 \lambda^{2} R_{1}^{2} A^{-1}\left[\hat{J}_{m 1}+\left(\lambda^{2}+\sigma_{2}\right) \hat{J}_{m 0}\right]
\end{gathered}
$$

in which $\hat{I}_{m 2}$ is the $I_{m 2}$ in (I.79), with $u_{1}$ changed to $\hat{u}_{1}, \hat{J}_{m 1}$ is the $J_{m 1}$ in (I.79), with $v_{1}$ changed to $\hat{v}_{1}$, and similarly for the other integrals. Similarly, multiplying (36) by $r \hat{u}_{1}$ and integrating, we obtain

$$
\begin{gathered}
\hat{I}_{m 2}+\left(2 \lambda^{2}+\sigma_{2}\right) \hat{I}_{m 1}+\lambda^{2}\left(\lambda^{2}+\sigma_{2}\right) \hat{I}_{m 0}-b D \hat{u}_{1}(b) L u_{2}(b) \\
=-4 \lambda^{2} R_{2}^{2} A^{-1}\left[\hat{J}_{m 1}+\left(\lambda^{2}+\sigma_{1}\right) \hat{J}_{m 0}\right] .
\end{gathered}
$$

The difference of (38) and (39) is

$$
\begin{gathered}
\left(\sigma_{2}-\sigma_{1}\right)\left(\hat{I}_{m 1}+\lambda^{2} \hat{I}_{m 0}-4 \lambda^{2} A^{-1} R_{1}^{2} \hat{J}_{m 0}\right)+b D u_{2}(b) L \hat{u}_{1}(b)-b D \hat{u}_{1}(b) L u_{2}(b) \\
=-4 \lambda^{2} A^{-1}\left(R_{2}^{2}-R_{1}^{2}\right)\left[\hat{J}_{m 1}+\left(\lambda^{2}+\sigma_{1}\right) \hat{J}_{m 0}\right] .
\end{gathered}
$$

For convenience we define

$$
\begin{aligned}
& K=\int \frac{1}{r} D(r \hat{v}) D(r v)+\left(\lambda^{2}+\sigma\right) \int r \hat{v} v, \\
& M=\int \frac{1}{r} D(r \hat{u}) D(r u)+\lambda^{2} \int r \hat{u} u-4 \lambda^{2} A^{-1} R^{2} \int r \hat{v} v .
\end{aligned}
$$

If $b D u(b)$ and $b D \hat{u}(b)$ are kept at $\mp 1$ and $R^{2}$ is fixed, the limiting form of (40) is

$$
\frac{d}{d \sigma} L \bar{u}(b)=\mp M
$$

in which $d L \bar{u}(b)$ denotes $L u(b, \sigma+d \sigma)-L \hat{u}(b, \sigma)$ * $^{\star}$ The notation is rather awkward, but the concept is useful. Similarly, if $b L u(b)$ and $b L \hat{u}(b)$ are kept at \pm 1 and $R^{2}$ is kept constant, we have

$$
\frac{d}{d \sigma} D \bar{u}(b)=\mp M,
$$

with $d D \bar{u}(b)$ similarly defined. If $\sigma$ is kept constant, the formulae corresponding to (43) and (44) are

$$
\frac{d}{d R^{2}} L \bar{u}(b)=\mp 4 \lambda^{2} A^{-1} K \quad \text { for } b D u(b)=b D \hat{u}(b)=\mp 1
$$

and

$$
\frac{d}{d R^{2}} D \ddot{u}(b)=\mp 4 \lambda^{2} A^{-1} K \quad \text { for } b L u(b)=b L \hat{u}(b)= \pm 1 \text {. }
$$

It is interesting and important that if $D u(b)$ or $L u(b)$ vanishes for a pair of eigenvalues $\left(R^{2}, \sigma\right)$, so does $D \hat{u}(b)$ or $L \hat{u}(b)$.

\footnotetext{
* or $L \hat{u}(b, \sigma+d \sigma)-L u(b, \sigma)$, as the case may be.
} 
Combining (43) and (45), we have, along an $R^{2}-\sigma$ curve of the auxiliary system,

$$
\frac{d R^{2}}{d \sigma}=-\frac{A M}{4 \lambda^{2} K} \quad \text { for } b D u(b)=\mp 1=b D \hat{u}(b) .
$$

If we eliminate $\hat{v}$ between (31) and (32), one single equation in $\hat{u}$ results, which is exactly the same (with $\hat{u}$ replacing $v$ ) as (I.10), with exactly the same boundary conditions as far as the auxiliary system is concerned. Hence $\hat{u}$ is proportional to $v$; the constant of proportionality changes sign only at $R^{2}=0$, i.e., at $\sigma_{n 0}^{\prime \prime}$ of Set I and Set II. Since

$$
K=-\int A\left[1-\varepsilon B\left(1-\frac{1}{r^{2}}\right)\right] v \hat{u}
$$

(the limits of integration here are 1 and $b$, and the conventional $d r$ is again omitted), it is evident that $K$ is nonzero except at $R^{2}=0$ for the auxiliary system, since the bracket in the integrand is positive in $0 \leqq \varepsilon \leqq 1$ and $\hat{u}$ is proportional to $v$. This fact makes it impossible for two branches of the $R^{2}-\sigma$ curves of the auxiliary system to merge smoothly into one curve at $R^{2}>0$, as will now be shown.

Suppose that (in the stated range of $\varepsilon$ ) a merging of two * branches occurs at $\varepsilon=\varepsilon_{0}$. Then for $\varepsilon$ slightly greater than $\varepsilon_{0}$, there is at least one extremum (in fact, two extrema) of $R^{2}$ where $M$ is zero. For $\varepsilon=0$ we have $u=\hat{u}$ and $v=\hat{v}$, and hence $M>0$ everywhere. Thus by continuity $M$ is positive everywhere along the merging curve except at the point of merging. Thus $M$ has at least a double zero at this point, which (by virtue of (47)) then cannot be the point of merging of two branches with $K$ nonzero for $R^{2}>0$. This leads to a contradiction. Hence for the auxiliary system there is no maximum or minimum of $R^{2}$ if $R^{2}$ is positive, which means that all the eigenvalues of $\sigma$ remain real for non-negative $R^{2}$. We also recall that if $\sigma$ is real the eigenvalues of $R^{2}$ for the auxiliary system are real. Hence we have proved

Theorem 5. For the auxiliary system and any $\varepsilon$ in $0 \leqq \varepsilon \leqq 1$, the eigenvalues of $R^{2}$ are real if $\sigma$ is real, and the eigenvalues of $\sigma$ are real for non-negative $R^{2}$. The $R^{2}-\sigma$ curves are therefore monotonic for positive $R^{2}$, the $n$-th curve possessing two branches which merge at $\sigma=\sigma_{n 0}^{\prime \prime}$ and $R^{2}=0$. The curves are therefore all parabolalike.

The next task is to show that $M$ is always positive for positive $R^{2}$. We have seen that it is positive at $\varepsilon=0$. If $M=0$ at any point on an $R^{2}-\sigma$ curve of the auxiliary system for $\varepsilon>0$ and $R^{2}>0$, there would be a triple eigenvalue of $\sigma$ there, giving rise to two extraneous (and complex) eigenvalues of $\sigma$. This is true whether the point in question is a simple point or a multiple point where two (or more) $R^{2}-\sigma$ curves meet but do not merge. Hence we have

Theorem 6. For positive $R^{2}$ the value $M$ for the auxiliary system is positive.

We note that for any system the multiple points cannot constitute a dense set, or even a set of infinite points with a finite limit point, for otherwise two $R^{2}-\sigma$ curves of that system would collapse into one (coinciding with an $R^{2}-\sigma$

* Merging of three or more branches must be preceded by mergings of two branches. 
curve of a neighboring system) and this would lead to a contradiction as we continue the curves to the $\sigma$-axis. [The assertion that if multiple points were not isolated then two $R^{2}-\sigma$ curves would collapse is based on the theorem that two analytic functions are identical if they are equal at an infinite set of points containing a finite limit point, and on the fact that $R^{2}$ is an analytic function of $\sigma$ along any $R^{2}-\sigma$ curve.]

\section{The $R^{2}-\sigma$ Curves for the Intermediate System for $0 \leqq \varepsilon \leqq 1$}

For any positive $R^{2}$ and for the intermediate system, we shall prove the existence of real eigenvalues of $\sigma$, and real ones only, by an evolutional procedure based on (43) and (44). For any positive $R^{2}$ we start with a value of $\sigma$ large enough that to the right of it there are no eigenvalues of $\sigma$. We can always start with a positive $\sigma$ at which $R^{2}$ is less than $E_{1}^{\prime \prime}$, the smallest eigenvalue of $R^{2}$ for the auxiliary system. Then either $b D \hat{u}(b) L u(b)$ or $b D u(b) L \hat{u}(b)$ is initially positive. We can keep $b D \bar{u}(b)$ (i.e., $b D u(b)$ or $b D \hat{u}(b)$ ) at the value -1 and decrease $\sigma . L \bar{u}(b)$ (i.e., $L \hat{u}(b)$ or $L u(b))$ is then initially negative. Since eigenvalues of $\sigma$ for the auxiliary system do exist, $L \vec{u}(b)$ will increase to zero as $\sigma$ decreases to the largest eigenvalue. (Now we do not need to distinguish which set it belongs to, and order the eigenvalues $\sigma_{n}^{\prime \prime}$ in one series of decreasing value.) As $\sigma$ decreases further, $L \bar{u}(b)$ will be positive. Keeping $b L \bar{u}(b)$ at the value 1 and decreasing $\sigma$ further, we assert that $D \bar{u}(b)$ must change sign at some $\sigma$, for otherwise we could keep $b D \bar{u}(b)$ at -1 and decrease $\sigma$. Then, since $M$ is positive for the auxiliary system, according to (43) $L \bar{u}(b)$ could never be zero again. To see this, note that $L \bar{u}(b)$ is positive after $\sigma$ passes $\sigma_{1}^{\prime \prime}$, and that, since $M$ is positive, it would have to increase to zero at $\sigma_{2}^{\prime \prime}$, which is impossible. Hence $D \bar{u}(b)$ must change sign at least once between $\sigma_{1}^{\prime \prime}$ and $\sigma_{2}^{\prime \prime}$, at a value which we denote by $\sigma_{1}^{\prime}$. Similarly it vanishes at least once between two consecutive eigenvalues of $\sigma$ of the auxiliary system. It cannot vanish more than once, for otherwise there would be an extraneous eigenvalue as $R^{2}$ decreases to zero. Hence all the eigenvalues of $\sigma$ are real, and we have proved

Theorem 7. The $R^{2}-\sigma$ curves of the intermediate system separate those of the auxiliary system for non-negative $R^{2}$ and for $0 \leqq \varepsilon \leqq 1$, and vice versa. Therefore all the eigenvalues of $\sigma$ for the intermediate system are real for non-negative $R^{2}$.

The process of evolution leading to Theorem 7 clearly shows that, except at multiple points (of either the auxiliary system or the intermediate system), if $D \bar{u}(b)$ vanishes then $L \bar{u}(b)$ does not vanish, and vice versa. Thus at a simple point on an $R^{2}-\sigma$ curve of the intermediate system which does not happen to be a multiple point of the auxiliary system, the value $L \bar{u}(b)$ is nonzero and (44) is valid. The fact that the point is simple then implies that $M$ is nonzero, for otherwise the point would be a multiple one for the intermediate system, by virtue of (44). Since these simple points may be joined to a point on an $R^{2}-\sigma$ curve of the same system at $\varepsilon=0$ by a curve consisting entirely of simple points, it follows that $M$ must be positive at any simple point if it cannot vanish at any simple point. Thus we have shown

Theorem 8. $M$ is positive along $R^{2}-\sigma$ curves of the intermediate system except possibly at the multiple points of that system or of the auxiliary system. 
Actually we can prove that $M$ is positive even at multiple points. Since the proof is long and since we do not need this result for what follows, we shall omit it.

\section{The $R^{2}-\sigma$ Curves for the Original System}

Since multiple points are isolated, the $R^{2}$ values at which they are located are not only of measure zero but without a finite limit point. This makes it possible to ignore multiple points altogether. After we have obtained the result for nonnegative values of $R^{2}$ less this countable set, the conclusion automatically applies to all non-negative values of $R^{2}$, by virtue of continuity.

By demanding that

$$
\bar{u}(1)=\bar{v}(1)=0=\bar{v}(b)=\bar{u}(b)=D \bar{u}(b)
$$

where $\bar{u}$ is $u$ or $\hat{u}, \bar{v}$ is $v$ or $v$, by fixing $R^{2}$ at a positive value, and by decreasing $\sigma$ from a sufficiently large initial value, we can, using arguments similar to those in Section 5, reach the eigenvalues of the intermediate system and the transitory system alternately. Thus we have proved

Theorem 9. The $R^{2}-\sigma$ curves of the transitory system separate and are separated by those of the intermediate system for non-negative $R^{2}$ and for $\varepsilon$ in the interval $0 \leqq \varepsilon \leqq 1$. Therefore all the eigenvalues of $\sigma$ for the transitory system are real for non-negative $R^{2}$.

Since Theorem 9 contains the statement that for non-negative $R^{2}$ the eigenvalues of $\sigma$ for the original system are real, we have reached our principal goal.

By the arguments leading to Theorem 8 , we can also prove

Theorem 10. $M$ is positive along the $R^{2}-\sigma$ curves of the transitory system and, in particular, of the original system, except possibly at the multiple points of that system or of the intermediate system.

We note here that Theorem 13 of Part I and Theorem 9 of this part are based on the implicit understanding that new eigenvalues (possibly complex) of $R^{2}$ for fixed $\sigma$, or of $\sigma$ for fixed $R^{2}$, cannot enter at infinity as $\varepsilon$ increases from zero. This entrance of eigenvalues at infinity can be ruled out by an asymptotic solution for large $\left|R^{2}\right|$ or large $|\sigma|$. An asymptotic analysis shows that, when $\left|R^{2}\right|$ is very large, $R^{2}$ is real and positive, and that when $|\sigma|$ is very large, $\sigma$ is real and negative and equal to the eigenvalues of $\sigma$ when $|\sigma|$ is very large and $R^{2}=0$. Hence the eigenvalues of $R^{2}$ for every large $\left|R^{2}\right|$ and of $\sigma$ for very large $|\sigma|$ are just the ones we have considered, and there can be no entry of new ones at infinity.

The proof of the assertion for the case of large $|\sigma|$ is obvious. The assertion for the case of large $\left|R^{2}\right|$ bears on Theorem 13 of Part I; its truth is not required for Theorem 9 in this part. The proof that $R^{2}$ is real and positive for large $\left|R^{2}\right|$ for any of the three systems (and, in particular, for the original system) depends on comparing the system (a) consisting of

$$
\begin{aligned}
L^{2} u & =4 \lambda^{2} R^{2}[f(r)]^{2} v \\
L v & =A u
\end{aligned}
$$


and the boundary conditions, with the system $(b)$ consisting of

$$
\begin{aligned}
L^{2} u & =4 \lambda^{2} R^{2} f(r) v, \\
L v & =A f(r) u,
\end{aligned}
$$

and the boundary conditions, with $f(r)$ defined by

$$
[f(r)]^{2}=1-\varepsilon B\left(1-\frac{1}{r^{2}}\right) .
$$

It can easily be shown that (i) the eigenvalues of $R^{2}$ for System (a) and those for System $(b)$ are asymptotically the same for very large $\left|R^{2}\right|$, and (ii) the eigenvalues of $R^{2}$ are real and positive for System $(b)$.

\section{Recapitulation of Results for Positive $\sigma+\lambda^{2}$}

At the intersection of two $R^{2}-\sigma$ curves of any one system there are two independent modes of that system, with two independent eigenfunctions for $u$, which can be combined linearly to satisfy the boundary conditions of a neighboring system. Hence an $R^{2}-\sigma$ curve of the neighboring system must pass through such an intersection point.

We assert that, for non-negative $\sigma+\lambda^{2}$, two $R^{2}-\sigma$ curves of the auxiliary system cannot intersect. For if they did there would be a mode with eigenfunctions $u$ and $v$ satisfying the boundary conditions of both the auxiliary system and the intermediate system, which would contradict the results given in the paragraph containing (I.66). For the same reason, two $R^{2}-\sigma$ curves of the intermediate system or the transitory system cannot intersect.

Since $R^{2}-\sigma$ curves of one system are separated by those of a neighboring system, an $R^{2}-\sigma$ curve of one system cannot intersect one of a neighboring system. For to preserve the separation, a third curve (of one or the other system) would have to pass through the point of intersection, and this contradicts the remarks of the preceding paragraph. Hence $R^{2}-\sigma$ curves of neighboring systems are completely separated, with no intersections whatever.

We also recall that the operators $M$ and $K$ of the auxiliary system are both positive for non-negative $\lambda^{2}+\sigma$ (see Theorem 6 and the discussion following (48)). From (47) we see that for the auxiliary system $R^{2}$ increases with $\sigma$, i.e., the $R^{2}-\sigma$ curves are ascending. Now consider the $R^{2}-\sigma$ curves of the intermediate system; they must also be ascending since they are separated by those of the auxiliary system, and since on each of them $R^{2}$ is a single-valued function of $\sigma$, and vice versa. Similarly, the $R^{2}-\sigma$ curves of the transitory system (and, in particular, the original system) are ascending. That is, for neutral or unstable Taylor vortices $(\sigma \geqq 0) \sigma$ increases with $R^{2}$ for each mode.

\section{The Main Theorem}

With the results of Section 7 in mind, we may summarize the results of Theorem 10 of Part I and Theorem 9 of this part, as follows:

Main Theorem. Taylor vortices in an incompressible fluid between cylinders rotating in the same direction and with the circulation decreasing outward are 
necessarily nonoscillatory, whether stable or unstable. For a given non-negative $R^{2}$ there are infinitely many real eigenvalues $\sigma_{n}$, some of which may be positive. When $\sigma$ is positive, the $u$ and $v$ for the nth mode have $n-1$ internal zeros, and the growth rate $\sigma$ increases with $R^{2}$ for each mode. Among the unstable modes for any given (non-negative) $R^{2}$, the lower the mode, the more unstable it is.

This theorem is our main conclusion. We remark that a spectral theory for Bénard cells with a monotonically decreasing nonlinear temperature can be constructed along exactly the same lines with (mutandis mutatis) exactly the same Main Theorem.

Acknowledgement. This work has been supported mainly by the National Science Foundation and the Office of Naval Research. The revision was partly carried out during my stay at the University of Grenoble; I wish to express my appreciation to my colleagues there, and especially to Professor A. CRAYA, for their hospitality. I also want to express my gratitude to my friends who have encouraged me in this work.

Department of Engineering Mechanics University of Michigan Ann Arbor 